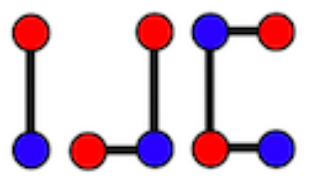

\title{
Relationship between adjacency and distance matrix of with diameter two
}

\author{
Siti L. Chasanah ${ }^{\mathrm{a}}$, Elvi Khairunnisa ${ }^{\mathrm{b}}$, Muhammad Yusuf ${ }^{\mathrm{b}}$, Kiki A. Sugeng ${ }^{\mathrm{b}}$ \\ aDepartment of Mathematics, Faculty of Mathematics and Natural Sciences, Universitas Lampung, \\ Bandar Lampung 35141, Indonesia \\ ${ }^{b}$ Department of Mathematics, Faculty of Mathematics and Natural Sciences, Universitas Indonesia, \\ Kampus UI Depok, Depok 16424, Indonesia
}

schasanah93@fmipa.unila.ac.id, elvikhairunnisa@gmail.com, yusuf.kappas@gmail.com, kiki@sci.ui.ac.id

\begin{abstract}
The relationship among every pair of vertices in a graph can be represented as a matrix, such as in the adjacency matrix and the distance matrix. Both adjacency and distance matrices have the same property. Adjacency and distance matrices are both symmetric matrices with diagonals entries equals to 0 . This paper discusses relationships between the adjacency matrix and the distance matrix graph of diameter two, which is $D=2(J-I)-A$. From this relationship, we also determine the value of determinant matrix $(A+D)$ and lower bound of determinant of distance matrix of a graph of diameter two.
\end{abstract}

Keywords: adjacency matrix, distance matrix, diameter two

Mathematics Subject Classification: 05C12, 05C50

DOI: $10.19184 /$ ijc.2021.5.2.1

\section{Introduction}

A graph $G$ is defined as a pair of set $(V, E)$ where $V$ is a non-empty finite set of objects (called as the vertices of $G$ ) and $E$ is the set (possibly empty) of unordered pairs of different vertices in $V$ (called as the edge of $G$ )[2]. The adjacent relationship of vertices in a graph can be represented in a matrix with ordo $n \times$, called an adjacency matrix. The rows and columns of this matrix

Received: 4 June 2018, Revised: 4 May 2021, Accepted: 15 September 2021. 
represent vertices, and entries of this matrix represent a relationship between vertices. We denote the adjacency matrix with $A=A(G)$ in which the $i j$-entry is 1 if there is an edge $i j$ from vertices $i$ to $j$, and 0 otherwise [1].

Distance matrix $D=\left(d_{i j}\right)$ of a graph $G$ has entries $d_{i i}=0$ and $d_{i j}$ is equal to the length of the shortest path between the vertices $i$ and $j$ [5]. This matrix has the same property as the adjacency matrix, such that it is a symmetric matrix with diagonal entries are all 0 . The maximum length between two vertices in a graph $G$ is called the diameter, notated by diam $(G)$ [2]. The graph discussed in this paper is a simple graph with diameter two. The graph with a diameter two is chosen since this graph is the simplest graph explaining the relationship between the adjacency matrix and the distance matrix. Some examples of graphs with diameter two that are familiar are complete bipartite graphs, wheel graphs, friendship graphs, and fan graphs.

This paper discusses the relationship between the adjacency matrix and the distance matrix of a graph of diameter two. Furthermore, we gave the value of $\operatorname{det}(A+D)$ and lower bound of $\operatorname{det}(D)$.

\section{New Results}

Theorem 2.1. Let $G$ be a graph with diameter two, $D$ be its distance matrix, and $A$ is its adjacency matrix, then $D=2(J-I)-A$, which $J$ is a matrix with all entries are 1 and $I$ is an identity matrix.

Proof. Let $G$ be a graph with diameter two and $D=\left(d_{i j}\right)$ be a distance matrix with $d_{i j}$ is the length of the shortest path between the vertices $i$ and $j$. Thus, we obtain the entries of a matrix $D$ as follows.

$$
d_{i j}= \begin{cases}0, & \text { if } i=j \\ 1, & \text { if } i \text { and } j \text { is adjacent } \\ 2, & \text { if } i \text { and } j \text { is not adjacent. }\end{cases}
$$

Let $A=\left(a_{i j}\right)$ be an adjacency matrix. Define

$$
\begin{aligned}
X & =2(J-I)-A \\
= & {\left[\left(\begin{array}{cccc}
1 & 1 & \cdots & 1 \\
1 & 1 & \cdots & 1 \\
\vdots & \vdots & \ddots & \vdots \\
1 & 1 & \cdots & 1
\end{array}\right)-\left(\begin{array}{cccc}
1 & 0 & \cdots & 0 \\
0 & 1 & \cdots & 0 \\
\vdots & \vdots & \ddots & \vdots \\
0 & 0 & \cdots & 1
\end{array}\right)-A\right] } \\
= & \left(\begin{array}{cccc}
0 & 2 & \cdots & 2 \\
2 & 0 & \cdots & 2 \\
\vdots & \vdots & \ddots & \vdots \\
2 & 2 & \cdots & 2
\end{array}\right)-A
\end{aligned}
$$

with $a_{i j}= \begin{cases}0, & \text { if } i=j \text { or } i \text { and } j \text { is not adjacent, } \\ 1, & \text { if } i \text { and } j \text { is adjacent. }\end{cases}$ 
Let $Y=\left(y_{i j}\right)=\left(\begin{array}{cccc}0 & 2 & \cdots & 2 \\ 2 & 0 & \cdots & 2 \\ \vdots & \vdots & \ddots & \vdots \\ 2 & 2 & \cdots & 2\end{array}\right)$, then we obtain entries of matrix $\left(x_{i j}\right)=\left(y_{i j}\right)-\left(a_{i j}\right)$ are as follows.

$$
x_{i j}= \begin{cases}0, & \text { if } i=j, \\ 1, & \text { if } i \text { and } j \text { are adjacent, } \\ 2, & \text { if } i \text { and } j \text { are not adjacent. }\end{cases}
$$

Since the size and entries of matrix $X$ and $D$ are the same, then it is proven that $X=D=$ $2(J-I)-A$.

Lemma 2.1. Given the $n \times n$ matrix $J=\left(e_{i j}\right)$, where $e_{i j}=1$ for all $i, 1<i<n$, and all $j$, $1<j<n$, then

$$
J^{2}-J=(n-1) J
$$

Proof. Since $e_{i j}=1$, then

$$
\begin{aligned}
e_{i j} \cdot e_{i j} & =[J]_{i, w} \cdot[J]_{w, i} \\
& =\sum_{w=1}^{n}(1)(1) \\
& =\sum_{w=1}^{n} 1 \\
& =n .
\end{aligned}
$$

Then $J^{2}-J$ is a matrix of order $n \times n$ with all entries are $n-1$ or can be write as $J^{2}-J=$ $(n-1) J$.

Corollary 2.1. Let $A$ be an adjacency matrix with diameter of two, $D$ be a distance matrix, and $J=\left(e_{i j}\right)$ be a matrix of order $n \times n$, where $e_{i j}=1$ then

$$
A J=2(n-1) J-D J
$$

Proof. From Theorem 1.1., we know that $A=2(J-I)-D$.

$$
\begin{aligned}
A J & =(2(J-I)-D) J \\
& =(2 J-2 I-D) J \\
& =2\left(J^{2}-J\right)-D J
\end{aligned}
$$

Based on Lemma 2.1, we knew that $J^{2}-J=(n-1) J$. Thus it is proven that $A J=2(n-1) J-$ $D J$.

Theorem 2.2. Let $A$ be an adjacency matrix and $D$ be a distance matrix of a graph $G$ of order $n$, then

$$
\operatorname{det}(A+D)=2^{n}(-1)^{n-1}(n-1) .
$$


Proof. Based on Theorem 2.1., we have $D=2(J-I)-A$. Thus $D+A=2(J-I)$. $\operatorname{det}(A+D)$ can be obtained by elementary row operations on the matrix $A+D$.

$$
\operatorname{det}(A+D)=\left(\begin{array}{ccccccccc}
0 & 2 & 2 & 2 & 2 & 2 & \cdots & 2 \\
2 & 0 & 2 & 2 & 2 & 2 & \cdots & 2 \\
2 & 2 & 0 & 2 & 2 & 2 & \cdots & 2 \\
2 & 2 & 2 & 0 & 2 & 2 & \cdots & 2 \\
2 & 2 & 2 & 2 & 0 & 2 & \cdots & 2 \\
2 & 2 & 2 & 2 & 2 & 0 & \cdots & 2 \\
\vdots & \vdots & \vdots & \vdots & \vdots & \vdots & \ddots & \vdots \\
2 & 2 & 2 & 2 & 2 & 2 & \cdots & 0
\end{array}\right)
$$

Using elementary row operation, we have the following result.

$$
\begin{aligned}
\operatorname{det}(A+D) & =2^{n}(-1)^{n-1}\left(\begin{array}{cccccccc}
1 & 0 & 1 & 1 & 1 & 1 & \cdots & 1 \\
0 & 1 & 1 & 1 & 1 & 1 & \cdots & 1 \\
0 & 0 & 1 & 2 & 1 & 1 & \cdots & 1 \\
0 & 0 & 0 & 1 & -1 & 0 & \cdots & 1 \\
0 & 0 & 0 & 0 & 1 & -1 & \cdots & 0 \\
0 & 0 & 0 & 0 & 0 & 1 & \cdots & 0 \\
\vdots & \vdots & \vdots & \vdots & \vdots & \vdots & \ddots & \vdots \\
0 & 0 & 0 & 0 & 0 & 0 & \cdots & n-1
\end{array}\right) \\
& =2^{n}(-1)^{n-1}(n-1) .
\end{aligned}
$$

In [4] Ryser mentioned that the upper bound of the determinant of the square matrix which has entries 0 and 1 with size $n$ and $t$ of non-zero entries is as follows.

$$
\operatorname{det}(A) \leq\left(\frac{2 m}{n}\right)^{n}\left(1-\frac{2 m-n}{n(n-1)}\right)^{n-1} .
$$

Based on this formula, we obtain the lower bound of the distance matrix graph $\mathrm{G}$ with diameter two, aswestated in Theorem 2.3.

Theorem 2.3. Let $D$ be an $n \times n$ distance matrix of a graph $G$ with diameter two where $n \geq 2$ 
vertices and $m$ edges, then the lower bound of distance matrix $D$ is

$$
\operatorname{det}(D) \geq 2^{n}(-1)^{n-1}(n-1)-\left(\frac{2 m}{n}\right)^{n}\left(1-\frac{2 m-n}{n(n-1)}\right)^{n-1} .
$$

Proof. Since $A$ and $D$ are semidefinite positive matrices, then from Theorem 1.1, we obtain $\operatorname{det}(A+D) \geq \operatorname{det}(A)+\operatorname{det}(D)$.

According to inequality (*), we have $\operatorname{det}(A) \geq\left(\frac{2 m}{n}\right)^{n}\left(1-\frac{2 m-n}{n(n-1)}\right)^{n-1}$. Then

$$
\operatorname{det}(A+D) \geq \operatorname{det}(A)+\operatorname{det}(D) \leq\left(\frac{2 m}{n}\right)^{n}\left(1-\frac{2 m-n}{n(n-1)}\right)^{n-1}+\operatorname{det}(D) .
$$

By using Theorem 2.2., we obtain

$$
\operatorname{det}(D) \geq 2^{n}(-1)^{n}-1(n-1)-\left(\frac{2 m}{n}\right)^{n}\left(1-\frac{2 m-n}{n(n-1)}\right),
$$

with $n \geq 2$ vertices and $m$ edges.

\section{Conclusion}

Based on the results, we conclud that the relationship between the adjacency matrix and the distance matrix of a graph with a diameter two is $D=2(J-I)-A$. From this relationship, we can obtain the general form of the determinant of matrix $A+D$ and lower bound of the determinant of distance matrix of graph $\mathrm{G}$ with a diameter two.

\section{Acknowledgement}

Part of this research is funded by PUTI Saintekes No NKB-2412/UN2.RST/HKP.05.00/2020.

\section{References}

[1] R. Bapat, Graphs and Matrices, Hindustan Book Agency, Springer. 2010.

[2] G. Chatrand, L. Lesniak, P. Zhang, Graphs and Digraphs, 6th Edition.CRC Press. 2016.

[3] F. Harary, The determinant of the adjacency matrix of a graph. SIAM Review 4 (3) (1962), 202-210.

[4] H.J. Ryser, Maximal determinant in combinatorial investigation, Canad. J. Math., 8 (1978), 245-249.

[5] P. Krivka and N. Trinajstic, On the distance polynomial of graph, Aplikace Matematiky, 28(5) (1983), 357-363. 